\title{
PROGRESSIVE SHARE OF SECRET AUDIO BY CHINESE REMAINDER THEOREM AND INTEGER WAVELET TRANSFORM
}

\author{
Chien-Chang Chen \\ Tamkang University
}

No.151, Yingzhuan Rd., Tamsui Dist., New Taipei City 25137, Taiwan ccchen34@mail.tku.edu.tw

Jian-Ying Huang

Tamkang University

No.151, Yingzhuan Rd., Tamsui Dist., New Taipei City 25137, Taiwan canthony015@gmail.com

\section{ABSTRACT}

This study presents an $(s, t, n)$ progressive scheme for sharing a secret audio. In an $(s, t, n)$ progressive audio sharing scheme, $n$ shared audios are generated from the secret audio, gathering $s$ shared audios acquires coarse resolution of the secret audio, and using $t$ shared audios reconstructs the original secret audio losslessly. The Chinese Remainder Theorem is adopted in the proposed scheme to share filter results acquired from a 1-D integer wavelet transform with different thresholds for satisfying the progressive property. First, the maximum wavelet layer number needed in a 1-D integer wavelet transform is determined from thresholds $s$ and $t$. Then, the proposed scheme applies the secret audio to a 1-D integer wavelet transform for acquiring filter results under different layers. Lastly, all wavelet coefficients are partitioned to $(t-s+1)$ groups and share each group with different thresholds. The experimental results demonstrate that the proposed scheme can share secret audios efficiently and progressively.

Keywords: $(s, t, n)$ Thresholds, Secret Audio Sharing, Progressive, Chinese Remainder Theorem

\section{INTRODUCTION}

Multimedia data is popular in recent computer usage and therefore security with multimedia data is important. Secret sharing techniques deal with keeping essential multimedia data secretly. Conventional secret sharing methods share one secret data among participants, and an adequate number 
of shared data recovers the secret data. Most secret sharing techniques have been applied to images to acquire the secret image sharing schemes ${ }^{1,2,4,5,6,7}$, $8,11,12$. However, audio content is another popular type of multimedia data. Therefore, this paper studies the application of secret sharing strategies thru audio content.

Although this paper discusses a secret audio sharing scheme, previous studies on secret image sharing problems merit our review. Therefore, we first introduce important reviews on secret image sharing schemes. Since Thien and Lin $^{12}$ first presented a secret image sharing scheme, many methods for sharing secret images with different properties have been presented. These properties include fault tolerance ${ }^{1}$, progressive $e^{2,6,7}$, multi-sharing ${ }^{8}$, and stacking recovery ${ }^{4,5}$. Among these properties, there is progressive sharing containing the property of recovering the secret media with different quality according to the number of shared data. Gathering more shared data leads to better quality on recovered media. This property lets a user obtain media with different quality according to the number of shared data. As to schemes with the Chinese Remainder Theorem and audio content, Shen et al. ${ }^{10}$ discussed audio watermarking with the Chinese Remainder Theorem and this also provides us an efficient way to analyze audio.

This work presents a progressive sharing scheme for secret audio. Two topics are studied in this work. First, a method to acquire the progressive sharing property is important and this study uses a 1-D integer wavelet transform accompanied by different thresholds to satisfying the progressive sharing. Second, an efficient secret sharing scheme is important. Since an audio is a large 1-D data vector, an efficient scheme is needed to improve execution performance. In this study, the Chinese Remainder Theorem is adopted for its high efficiency. Consequently, this study presents a Chinese Remainder Theorem based $(s, t, n)$ scheme for progressively sharing a secret audio. An $(s, t, n)$ secret audio sharing scheme shares a secret audio among $n$ shared audios and $s$ shared audios are collected to recover the coarsest resolution of the secret audio. When collecting $t$ shared audios, the secret audio can be recovered losslessly. Thresholds $s$ and $t$ determine $(t-s+1)$ different resolutions in the progressive scheme.

The remainder of this paper is organized as follows. In Section 2, related topics including a 1-D integer wavelet transform, Chinese Remainder Theorem based secret sharing scheme, and PCM audio format are introduced. Section 3 illustrates the proposed progressive secret audio sharing method. Algorithms for sharing a secret audio among shared audios and recovering the secret audio from shared audios are presented in Sections 
3.1 and 3.2, respectively. Section 4 presents experimental results and comparisons. In Section 5, we draw our conclusions.

\section{REVIEWS ON RELATED TOPICS}

\subsection{PCM Audio Format}

This section introduces the Pulse-Code Modulation (PCM) audio format, which is the standard audio format used in computers. A digital audio is sampled from an analog signal to digital values by sampling rate and bit depth. A $44.1 \mathrm{KHz}$ sampling rate and 16 bits depth for each quantized value are digitized in a standard CD digital audio. Furthermore, two channels are also popularly used in stereo audio. Since each audio quantized value is determined from a continuous signal, neighboring values in PCM are highly correlated and apply transformations like a 1-D wavelet transform to efficiently filter coefficients belonging to different frequencies. This is the reason of our selection of a 1-D integer wavelet transform.

\subsection{1-D Integer Wavelet Transform}

This section introduces a 1-D integer wavelet transform to transfer a 1-D signal to wavelet coefficients ${ }^{3}$. Since the proposed scheme works on audio as large 1-D vector numbers, the computation load is an important consideration for the proposed scheme. Therefore, the proposed scheme selects the 1-D Haar wavelet based on its high efficiency.

Assume that a 1-D signal $\mathrm{S}$ denotes by $\left\{s_{0}, s_{1}, \ldots, s_{k-1}\right\}$, the low-pass filter results and high-pass filter results after performing a 1-D Haar wavelet transform as denoted by $L=\left\{l_{0}, l_{1}, \ldots, l_{k / 2-1}\right\}$ and $H=\left\{h_{0}, h_{1}, \ldots, h_{k / 2-1}\right\}$ are calculated by Eq. (1).

$$
\begin{aligned}
& h_{i}=s_{2 i}-s_{2 i+1} \\
& l_{i}=\left\lfloor\frac{s_{2 i}+s_{2 i+1}}{2}\right\rfloor
\end{aligned}
$$

The computed $l_{i}$ and $h_{i}(0 \leq i \leq k / 2-1)$ are integers and with high efficiency for our further sharing procedure by the Chinese Remainder Theorem. The inverse 1-D Haar wavelet transform acquires the original signal $S$ from $L$ and $H$ by Eq. (2).

$$
\begin{aligned}
& s_{2 i+1}=\left\lfloor\frac{2 l_{i}+1-h_{i}}{2}\right\rfloor \\
& s_{2 i}=h_{i}+s_{2 i+1}
\end{aligned}
$$




\subsection{Chinese Remainder Theorem Based $(t, n)$ Secret Sharing Scheme}

This section introduces the Chinese Remainder Theorem based $(t, n)$ secret sharing method ${ }^{9,10}$. First, $n$ ordered prime numbers $q_{i}\left(1 \leq i \leq n\right.$ and $q_{1}<$ $q_{2}<\ldots<q_{n}$ ) are needed to be determined. Then, two thresholds $\alpha$ and $\beta$, acquired from these prime numbers $q_{i}$, are calculated as products of prime numbers by $\alpha=\prod_{i=n-t+2}^{n} q_{i}<\prod_{i=1}^{t} q_{i}=\beta$. Each secret number should be shifted to a number between $\alpha$ and $\beta$ such that gathering $t$ shared numbers enables the recovery of the shifted number. However, collecting fewer than $t$ shared numbers will not obtain any recovered number exceeding $\alpha$. Therefore, no correct secret number can be acquired. Hence, the above shifting strategy satisfies $(t, n)$ thresholds in secret sharing problem.

\section{PROPOSED $(s, t, n)$ PROGRESSIVE SECRET AUDIO SHARING SCHEME}

This section introduces the proposed Chinese Remainder Theorem based $(s, t, n)$ progressive secret audio sharing scheme. The scheme generates $n$ shared audios for each participant, and collecting $s$ shared audios acquires a coarse version of the secret audio. Collecting more shared audios acquires finer recovered secret audio and collecting $t$ shared audios losslessly recovers the secret audio. Sections 3.1 and 3.2 present the sharing and recovery algorithms, respectively.

\subsection{Sharing Algorithm}

In an $(s, t, n)$ progressive secret audio sharing scheme, $n$ prime numbers $q_{i}(1 \leq i \leq n)$ are required. The secret key of a shared audio for participant $i$ is one prime number $q_{i}$. The sharing algorithm first transforms the secret audio to 1-D integer wavelet coefficients, in which the required transform layer is determined by thresholds $s$ and $t$. Assigning a different number of recovering thresholds to wavelet filter results provides the progressive property and these assignments are determined by thresholds parameters $s$ and $t$. The sharing algorithm is introduced as follows.

1. Determine the maximum wavelet layer $L_{\max }$ from thresholds $s$ and $t$ by Eq.(3)

$$
L_{\max }=t-s
$$

2. Apply the secret audio to 1-D integer wavelet transform, as noted in Eq. (1), to acquire wavelet low-pass filter result $L$ and high-pass filter results $H_{i}\left(1 \leq i \leq L_{\max }\right)$. 
3. Share $L$ to conventional $(s, n)$ secret sharing and $H_{i}\left(1 \leq i \leq L_{\max }\right)$ to conventional $(t-i+1, n)$ secret sharing by the following steps.

3.1. Randomly select $n$ prime numbers $q_{j}(1 \leq j \leq n)$ and $q_{j}$ has to fulfill the requirements of $q_{1}<q_{2}<\ldots<q_{n}$.

3.2 For sharing $L$, two parameters $\alpha$ and $\beta$ are needed with satisfying $\alpha<\beta$ by $\alpha=\prod_{j=n-s+2}^{n} q_{j}$ and $\beta=\prod_{j=1}^{s} q_{j}$. For sharing $H_{i}\left(1 \leq i \leq L_{\max }\right)$, two parameters $\alpha$ and $\beta$ are needed with satisfying $\alpha<\beta$ by $\alpha=\prod_{j=n-t+i+3}^{n} q_{j}$ and $\beta=\prod_{j=1}^{t-i+1} q_{j}$.

3.3. Partition one filter result to numbers $n_{k}$ and calculate $g_{k}$ using Eq.(4)

$$
g_{k}=n_{k}+\alpha+\gamma_{k}
$$

where $\gamma_{k}$ is a random number generated from one random seed.

3.4. Calculate the residue $r_{k}$ using Eq. (5)

$$
g_{k} \equiv r_{k}\left(\bmod q_{j}\right)
$$

3.5. Combine all $r_{k}$ to obtain the shared data from the wavelet filter result for participant $j$.

4. Combine all shared data and apply them to inverse wavelet transform, as noted in Eq.(2), to acquire the shared audio $S_{i}$ corresponding with participant's secret key $q_{j}$ for participant $j$.

Note that $\alpha$ used in Eq. (4) and the random seed to generate $\gamma_{k}$ should be stored in advance. Furthermore, the selected prime numbers $q_{j}$, noted as secret keys for each participant, are kept unchanged for sharing each filter result. Step 3 partitions $(t-s+1)$ wavelet filter results to different sharing and recovering thresholds. The progressive property is obtained from assigning $H_{i}$ to a $(t-i+1, n)$ secret sharing scheme. Therefore, each wavelet filter result, including $L$ and $H_{i}$, requires different recovery thresholds for the progressive property.

\subsection{Recovery Algorithm}

The secret audio can be progressively recovered from collecting $s$ shared audios to collecting $t$ shared audios using the recovery algorithm. The recovery algorithm for collecting $m(s \leq m \leq t)$ shared audios is introduced as follows. 
1. Collect $m(s \leq m \leq t)$ shared audios $S i(1 \leq i \leq m)$ with corresponding secret keys $p_{i}$ and apply each shared audio to the 1-D integer wavelet transform with a layer determined in Eq. (3) of the sharing algorithm.

2. Recover wavelet filter results $L$ and $H_{t-s}$ to $H_{t-m+1}$ of the original secret audio signal by the following steps.

2.1 Thresholds for the recovery of $L$ and $H_{j}(t-s \geq j \geq t-m+1)$ are defined by $k$ $(s \leq k \leq m)$, respectively.

2.2 For recovering a filter result with threshold $k$, we first calculate the shifted recovery number $r_{-} n_{j}$ using Eq. (6)

$$
\begin{gathered}
r_{-} n_{j}=\left(\sum_{i=1}^{k} n_{i, j} M_{i} M_{i}^{-1}\right) \bmod M \\
\text { where } M=\prod_{i=1}^{k} p_{i}, M_{i}=\frac{M}{p_{i}} \text {, and } M_{i}^{\prime}=M_{i}^{-1} \bmod p_{i} .
\end{gathered}
$$

2.3 Subtract the random number $\gamma_{j}$ and $\alpha$ from $r_{-} n_{j}$ to obtain the recovery numbers $m_{j}$.

$$
m_{j}=r_{-} n_{j}-\gamma_{j}-\alpha
$$

2.4 Collect $m_{j}$ to acquire one or more coefficients in the recovered wavelet filter result.

3. Collect all coefficients acquired in Step 2.4 to form one wavelet filter result and then apply all recovered wavelet filter results to inverse wavelet transform to acquire the recovered audio.

In Step 2, the recovered wavelet filter results are determined by thresholds $s, t$ and parameter $m$. If $s=m$, the recovery algorithm only recovers filter result $L$. If $s=m+1$, the recovery algorithm recovers $L$ and $H_{t-s}$. A larger $m$ recovers more high-pass filter results $H_{j}$. When $m=t, L$ and all $H_{j}(t-s \geq j \geq 1)$ are acquired to recover losslessly original secret audio. For $m>t$, using any $t$ shared audios is enough to acquire $L$ and all $H_{j}$ to recover the secret audio losslessly. Table 1 shows generated and recovered filter results under different parameters $s, t$, and $m$. The assignment of $s=2$ and $t$ $=4$ generates three different resolutions for recovered audios and they are different from recovering the progressive definitions $\{L\}$ or $\left\{L, H_{2}\right\}$ or $\{L$, $\left.H_{2}, H_{1}\right\}$, respectively. The parameter $m$ selects one of the progressive definitions. Furthermore, a larger $m$ value recovers more wavelet filter results and obtains a finer quality of the recovered audio. 
Table 1. Example of wavelet filter results under different parameters $s, t$, and $m$

\begin{tabular}{ccccc}
\hline$s$ & $t$ & $m$ & recovered filter results & generated filter results \\
\hline 2 & 4 & 2 & $L$ & $L, H_{2}, H_{1}$ \\
2 & 4 & 3 & $L, H_{2}$ & $L, H_{2}, H_{1}$ \\
2 & 4 & 4 & $L, H_{2}, H_{1}$ & $L, H_{2}, H_{1}$ \\
2 & 6 & 3 & $L, H_{4}$ & $L, H_{4}, H_{3}, H_{2}, H_{1}$ \\
2 & 6 & 5 & $L, H_{4}, H_{3}, H_{2}$ & $L, H_{4}, H_{3}, H_{2}, H_{1}$ \\
2 & 8 & 3 & $L, H_{6}$ & $L, H_{6}, H_{5}, H_{4}, H_{3}, H_{2}$, \\
& & & & $H_{1}$ \\
2 & 8 & 5 & $L, H_{6}, H_{5}, H_{4}$ & $L, H_{6}, H_{5}, H_{4}, H_{3}, H_{2}$, \\
\hline
\end{tabular}

\section{EXPERIMENTAL RESULTS AND COMPARISONS}

\subsection{Experimental Results}

This section presents the experimental results obtained from the proposed scheme. Programs of the proposed scheme were coded on an Android system and run on a mobile-phone with an ARM Cortex A9 CPU with $1 \mathrm{~GB}$ RAM and $1.4 \mathrm{GHz}$. The secret audio is a song of three seconds with almost 132,000 sampling points. Figure 1(a) depicts the signal amplitude of the secret audio. The thresholds $(s, t, n)$ are determined as $(2,3$, 4). This threshold assignment partitions the secret audio among four shared audios and two different-quality recovered secret audios can be acquired. The 1-D Haar integer wavelet transform, as introduced in Section 2.2, is calculated to transform the secret audio to wavelet filter results.

Figure 1 shows the experimental results of the proposed scheme. Figures 1(b)-(e) show four shared audios with secret keys of 257, 263, 269, and 271, respectively. Figure 1(f) shows the coarse recovered secret audio, which is acquired from any two shared audios. The recovered error of Figure 1(f), as defined by the difference between the recovered audio and the original secret audio, is depicted in Figure 1(h). The perfectly recovered secret audio, as shown in Figure 1(g), can be reconstructed using three or more shared audios. The recovered error of Figure 1(g), depicted in Figure 1(i), shows that the losslessly recovered audio in Figure 1(g).

Since the recovered audio from two shared audios is not the same as the original secret audio, the reconstructed error of using two shared audios and three shared audios are compared in Table 2. The reconstructed error, listed by Root Mean Square Error (RMSE), when using two shared audios is 878 and the reconstructed error when using three shared audios is the same as the original secret audio, which acquired a RMSE of 0. 


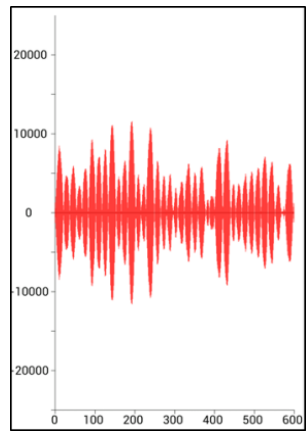

(a)

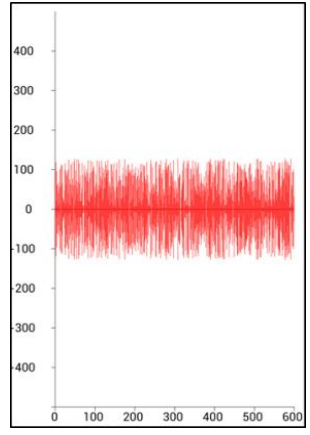

(d)

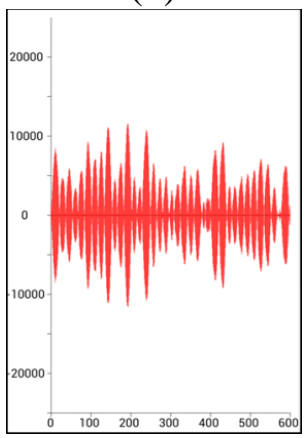

(g)

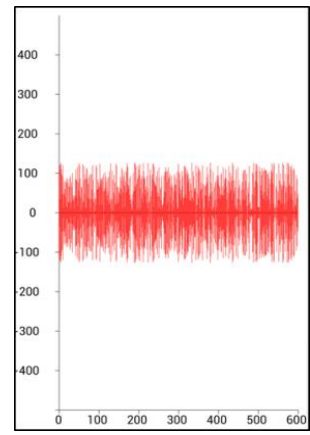

(b)

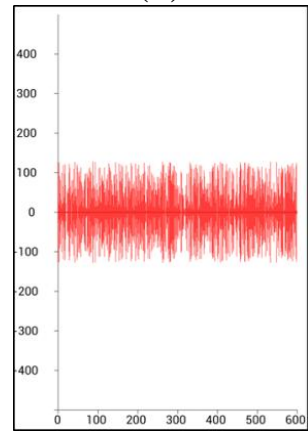

(e)

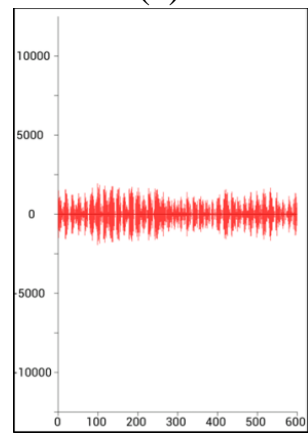

(h)

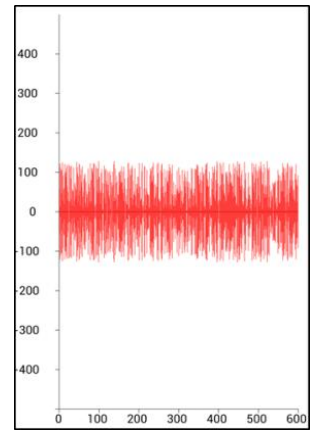

(c)

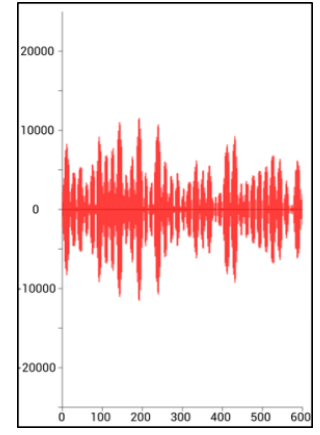

(f)

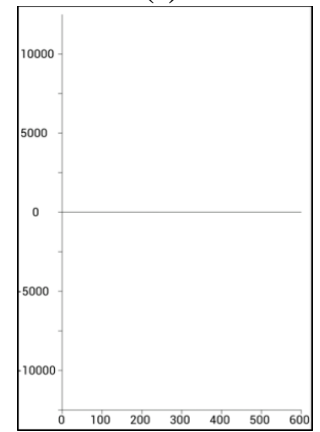

(i)

Figure 1. (a) Source secret audio, (b) shared audio with secret key 257, (c) shared audio with secret key 263, (d) shared audio with secret key 269, (e) shared audio with secret key 271, (f) recovered audio from two shared audios, (g) recovered audio from three shared audios, (h) recovered error of (f), (i) recovered error of (g)

Table 2. Root Mean Square Error (RMSE) of threshold (2, 3, 4).

RMSE of using two shared RMSE of using three shared audios audios 


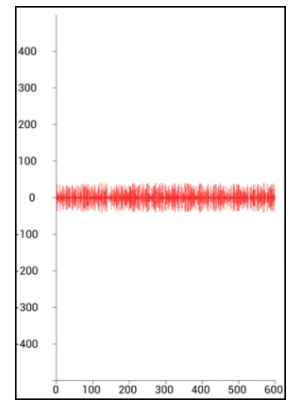

(a)

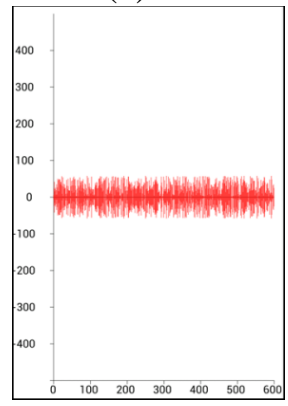

(e)

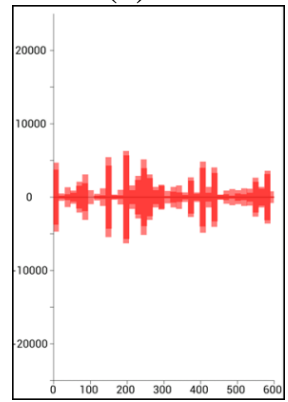

(i)

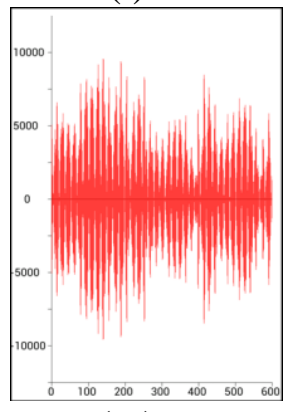

(m)

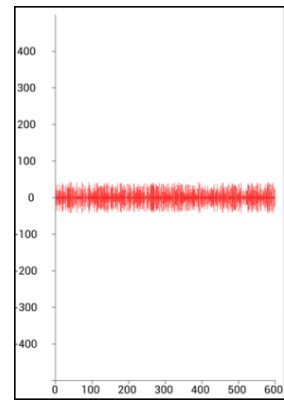

(b)

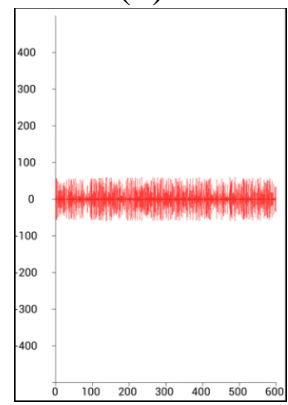

(f)

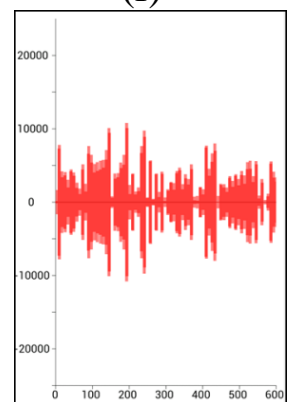

(j)

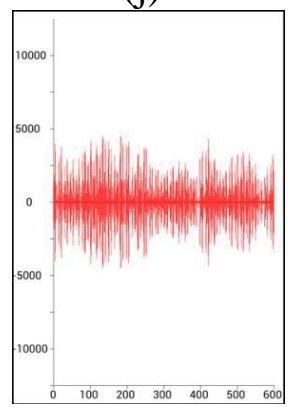

(n)

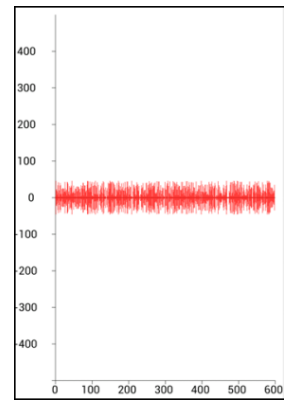

(c)

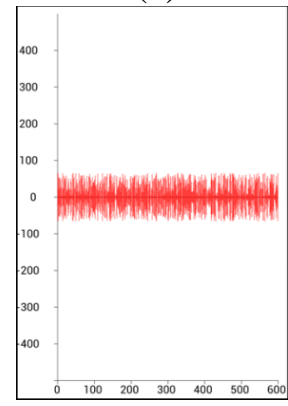

(g)

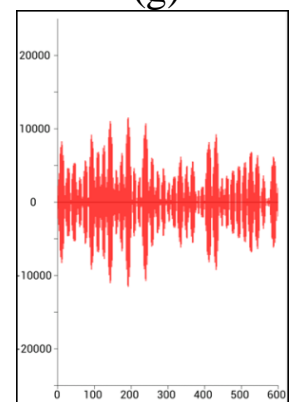

(k)

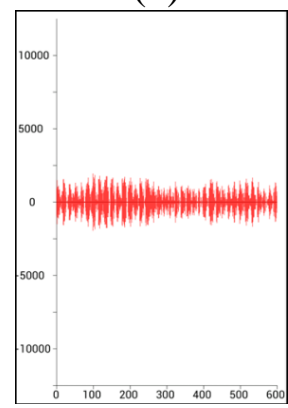

(o)

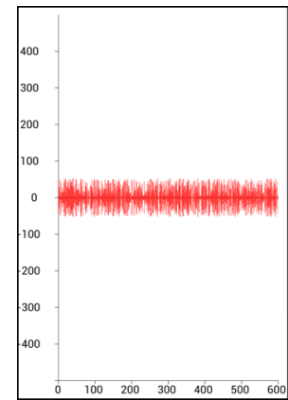

(d)

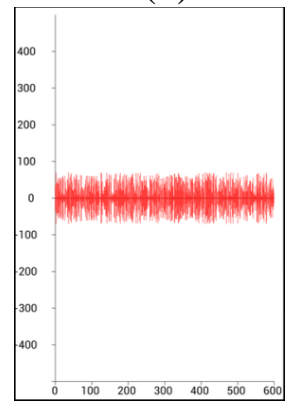

(h)

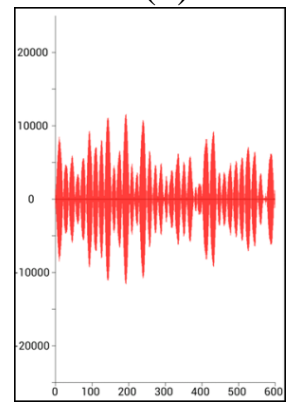

(1)

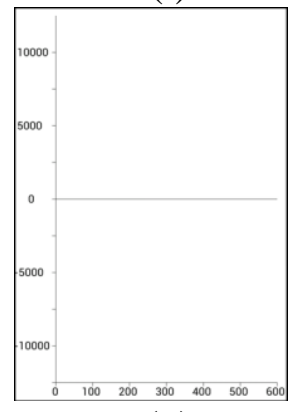

(p)

Figure 2. (a)-(h) shared audios of 8 different secret keys, (i) recovered audio from 3 shared audios, $(j)$ recovered audio from 4 shared audios, (k) recovered audio from 5 shared audios, (l) recovered audio from 6 shared audios, (m) recovered error of (i), (n) recovered error of (j), (o) recovered error of $(k),(p)$ recovered error of (l). 
Figure 2 shows the experimental results of other thresholds $(3,6,8)$ on the same secret audio used in Figure 1. Figures 2(a)-(h) show eight shared audios with secret keys of 41, 42, 47, 53, 59, 61, 67, and 71, respectively. Figure 2(i) shows the coarsest recovered secret audio, which is acquired from any three shared audios. The recovered error of Figure 2(i), as defined by the difference between the recovered audio and the original secret audio, is depicted in Figure 2(m). Figure 2(j) shows the recovered secret audio acquired from any four shared audios and the recovered error is depicted in Figure 2(n). Figure 2(k) shows the recovered secret audio acquired from any five shared audios and the recovered error is depicted in Figure 2(o). Figure 2(l) shows the recovered secret audio acquired from any six shared audios and the recovered error is depicted in Figure 2(p). From Figures 2(m)-(p), we find that recovered error is reduced according to the increasing number of recovered audios.

Table 3 lists the RMSE of threshold $(3,6,8)$. We can also find the reconstructed error of using more shared audios acquiring a lower RMSE value. Like experimental results in Table 2, using six shared audios shared audios acquired a RMSE value of 0 , which means that the recovered audio is the same as the secret audio.

Table 3. Root Mean Square Error (RMSE) of threshold $(3,6,8)$.

\begin{tabular}{ccccc}
\hline & $\begin{array}{c}\text { RMSE of } \\
\text { using three } \\
\text { hared audios }\end{array}$ & $\begin{array}{c}\text { RMSE of } \\
\text { using four } \\
\text { shared audios }\end{array}$ & $\begin{array}{c}\text { RMSE of } \\
\text { using five } \\
\text { shared audios }\end{array}$ & $\begin{array}{c}\text { RMSE of } \\
\text { using six } \\
\text { shared audios }\end{array}$ \\
\hline RMSE & 3,370 & 1,829 & 878 & 0 \\
\hline
\end{tabular}

\subsection{Comparisons}

Since there have been no previous studies on secret audio sharing, the proposed scheme can only make comparisons with progressive secret image sharing schemes. This section compares the proposed scheme with other progressive related works $1,2,4,5,6,7$, including visual cryptography and secret image sharing methods. The compared sharing features, as shown in Table 4, are explained as follows.

(1) Media content: Media content means the content for secret sharing and the compared media include digital image and digital audio. As listed in Table 4, all of the presented schemes are secret sharing for digital images. Only the proposed scheme studies sharing in digital audio.

(2) Sharing kernel: Sharing kernel lists the primary techniques to share media. Digital images always use a Shamir-Lagrange technique ${ }^{1,6,7}$ to 
share and recover losslessly. Sharing by visual block sharing strategy builds a visually similar image when recovering ${ }^{2,4,5}$.

(3) Lossless recovery: Not all methods can recover the original secret image losslessly. Only using mathematical methods like Shamir-Lagrange ${ }^{1,6}$ or Chinese Remainder Theorem can losslessly recover the secret media. Hung et al. $^{7}$ cannot recover losslessly because of DCT and embedding into cover images. Visual block sharing strategies use a stacking method to recover a visually similar secret image and this reconstruction cannot fulfill lossless recovery ${ }^{2,4}$, 5

(4) Progressive strategy: Each scheme listed in Table 4 uses its technique to accomplish a progressive property. Bit processing is the most popular method for progressive sharing ${ }^{1,2,4,5}$. Other methods and the proposed scheme use transforms, including the wavelet transform and DCT (Discrete Cosine Transform) to segment from coarse to fine resolutions of the secret image ${ }^{6,7}$.

Results in above figures and tables demonstrate that the proposed Chinese Remainder Theorem and the 1-D integer wavelet transform-based scheme performs efficiently when sharing an audio secretly. Comparisons also show that the proposed scheme is important for the secret presented audio sharing scheme.

Table 4. Comparisons between the proposed scheme and other progressive secret image sharing schemes $1,2,4,5,6,7$

\begin{tabular}{|c|c|c|c|c|}
\hline Schemes & $\begin{array}{l}\text { Media } \\
\text { content }\end{array}$ & Sharing kernel & $\begin{array}{l}\text { Lossless } \\
\text { recovery }\end{array}$ & $\begin{array}{c}\text { Progressive } \\
\text { strategy }\end{array}$ \\
\hline $\begin{array}{l}\text { Chen and } \\
\operatorname{Lin}^{1}\end{array}$ & $\begin{array}{l}\text { digital } \\
\text { image }\end{array}$ & Shamir-Lagrange & Yes & $\begin{array}{c}\text { Pixel bits } \\
\text { rearrangement }\end{array}$ \\
\hline Fang $^{2}$ & $\begin{array}{l}\text { digital } \\
\text { image }\end{array}$ & $\begin{array}{l}\text { Visual block } \\
\text { sharing }\end{array}$ & No & $\begin{array}{l}\text { Expanded block } \\
\text { replacement }\end{array}$ \\
\hline Hou et al. ${ }^{4}$ & $\begin{array}{l}\text { digital } \\
\text { image }\end{array}$ & $\begin{array}{l}\text { Visual block } \\
\text { sharing }\end{array}$ & No & $\begin{array}{l}\text { Recover with bit } \\
\text { shifting }\end{array}$ \\
\hline $\begin{array}{l}\text { Hou and } \\
\text { Quan } 5\end{array}$ & $\begin{array}{l}\text { digital } \\
\text { image }\end{array}$ & $\begin{array}{l}\text { Visual block } \\
\text { sharing }\end{array}$ & No & DCT coefficients \\
\hline $\begin{array}{l}\text { Huang et } \\
\text { al. }^{6}\end{array}$ & $\begin{array}{l}\text { digital } \\
\text { image }\end{array}$ & Shamir-Lagrange & Yes & wavelet SPIHT \\
\hline Hung et al. ${ }^{7}$ & $\begin{array}{l}\text { digital } \\
\text { image }\end{array}$ & Shamir-Lagrange & No & DCT frequency \\
\hline $\begin{array}{c}\text { The } \\
\text { proposed } \\
\text { scheme }\end{array}$ & $\begin{array}{l}\text { digital } \\
\text { audio }\end{array}$ & $\begin{array}{l}\text { Chinese } \\
\text { Remainder } \\
\text { Theorem }\end{array}$ & Yes & $\begin{array}{l}\text { 1-D wavelet filter } \\
\text { results }\end{array}$ \\
\hline
\end{tabular}




\section{CONCLUSIONS}

This study presented an $(s, t, n)$ progressive secret audio sharing scheme. The secret audio is progressively shared by assigning different thresholds to the audio's 1-D integer wavelet filter results. Different resolutions of the secret audio from coarse resolution, by collecting $s$ shared audios, to fine resolution, by collecting $t$ shared audios, are efficiently solved in the proposed scheme. Experimental results and discussions show that the proposed scheme does well in sharing a secret audio. Exploring different sharing strategies merits future study.

\section{REFERENCES}

[1] S.K. Chen, and J.C. Lin, Fault-tolerance and progressive transmission of images. Pattern Recognition, 38(12), p2466-2471, 2005. http://dx.doi.org/10.1016/j.patcog.2005.04.002.

[2] W.P. Fang, Friendly progressive visual secret sharing. Pattern Recognition, 41(4), p1410-1414, 2008. http://dx.doi.org/10.1016/j.patcog.2007.09.004.

[3] J.V. Fleet, Discrete wavelet transformations: An elementary approach with applications. Hoboken, N.J.: John Wiley \& Sons, 2008.

[4] Y.C. Hou, Z.Y. Quan, C.F. Tsai, and A.Y. Tseng, Block-based progressive visual secret sharing. Information Sciences, 233(1), p290-304, 2013. http://dx.doi.org/10.1016/j.ins.2013.01.006.

[5] Y.C. Hou, and Z.Y. Quan, Progressive visual cryptography with unexpanded shares. IEEE Trans. on Circuits and Systems for Video Technology, 21(11), p1760-1764, 2011. http://dx.doi.org/10.1109/TCSVT.2011.2106291.

[6] C.P. Huang, C.H. Hsieh, and P.S. Huang, Progressive sharing for a secret image. Journal of Systems and Software, 83(3), p517-527, 2010. http://dx.doi.org/10.1016/j.jss.2009.10.012.

[7] K.H. Hung, Y.J. Chang, and J.C. Lin, Progressive sharing of an image. Optical Engineering, 48(4), 047006, 2008. http://dx.doi.org/10.1117/1.2911719.

[8] S.J. Lin, S.K. Chen, and J.C. Lin, Flip visual cryptography (FVC) with perfect security, conditionally-optimal contrast, and no expansion. Journal of Visual Communication and Image Research, 21(8), p900-916, 2010. http://dx.doi.org/10.1016/j.jvcir.2010.08.006.

[9] M Quisquater, B Preneel, J. Vandewalla, On the security of the threshold scheme based on the Chinese remainder theorem. Paper presented at the 5th International Workshop on Practice and Theory in Public Key Cryptosystems, Paris, France, February 12-14, 2002. http://dx.doi.org/10.1007/3-540-45664-3_14.

[10] J.W. Shen, F. Pan, and Y. Guo, Digital Audio Watermark Sharing Based 
on Chinese Remainder Theorem. Paper presented at the 5th International Congress on Image and Signal Processing, China, October 16-18, 2012. http://dx.doi.org/10.1109/CISP.2012.6469667.

[11] S.J. Shyu, and Y.R. Chen, Threshold Secret Image Sharing by Chinese Remainder Theorem. In C.-H. Hsu, K.-C. Li, and B. Chapman (Ed.), Proceedings of the IEEE Asia-Pacific Services Computing Conference (p1332-1337). Yilan: IEEE Press, 2008. http://dx.doi.org/10.1109/APSCC.2008.223.

[12] C.C. Thien, and J.C. Lin, Secret image sharing. Computers \& Graphics, 26(5), p765-770, 2002. http://dx.doi.org/10.1016/S0097-8493(02)00131-0. 
